\title{
TV optimization and graph-cuts
}

\author{
J. Darbon ${ }^{1, *}$, S. Lefebvre ${ }^{2, * *}$, T.F. Chan ${ }^{3, * * *}$, and S. Esedoglu ${ }^{4, \dagger}$ \\ ${ }^{1}$ Mathematics Department of UCLA, Los Angeles, USA. \\ ${ }^{2}$ REVES /INRIA Sophia-Antipolis, France. \\ ${ }^{3}$ Mathematics Department of UCLA and NSF, Los Angeles, USA. \\ ${ }^{4}$ Mathematics Department, University of Michigan, Ann Arbor, USA.
}

This paper describes some links between the minimization of the Total Variation and the minimization of some binary energies.

(c) 2007 WILEY-VCH Verlag GmbH \& Co. KGaA, Weinheim

\section{The approach}

Assume that images are defined on a set of nodes $\mathcal{V}$ and let us denote by $u_{p} \in \mathcal{L}$ the value of the image $u$ at site $p \in \mathcal{V}$. Note that $\mathcal{L}$ will be defined later. It is assumed that the lattice is endowed with a neighborhood system and we consider the set of pairwise interactions $\mathcal{E}=\left\{(p, q) \in \mathcal{V}^{2}, p\right.$ and $q$ are neighbors $\}$. Given an observed image $v$ we wish to minimize the following energy:

$$
E(u \mid v)=\sum_{p \in \mathcal{V}}\left|u_{p}-v_{p}\right|+\sum_{(p, q) \in \mathcal{E}} w_{p q}\left|u_{p}-u_{q}\right|
$$

where $w_{p q}$ are some non-negative coefficients. Now we consider two different possibilities for $\mathcal{L}$, i.e., $\mathcal{L}=\{0,1\}^{2}=\mathcal{L}_{b}$ or $\mathcal{L}=\mathcal{R}=\mathcal{L}_{r}$. For the $\mathcal{L}_{b}$ case, the above optimization becomes a binary one. It has been shown [1,5] how to reduce this problem to the computation of a maximum flow on an associated graph. For the $\mathcal{L}_{r}$ case, we get the classical Total Variation (TV) minimization problem with $l^{1}$ fidelity. Now it has been observed in [2,3] that these two problems are closely related via the Co-area formula. Indeed the above energy rewrites as follows:

$$
E(u \mid v)=\int_{\mathcal{R}}\{\underbrace{\sum_{p \in \mathcal{V}}\left|\mathbb{1}_{u_{p} \leq \lambda}-\mathbb{1}_{v_{p} \leq \lambda}\right|+\sum_{(p, q) \in \mathcal{E}} w_{p q}\left|\mathbb{1}_{u_{p} \leq \lambda}-\mathbb{1}_{u_{q} \leq \lambda}\right|}_{E\left(\mathbb{1}_{u \leq \lambda} \mid v\right)}\} d \lambda .
$$

Approaches described in [3,4] to minimize the Total Variation states than one can minimize independently each binary subproblem $E\left(\mathbb{1}_{u \leq \lambda} \mid v\right)$. The latter can be done very efficiently with the maximum flow approach of [1,5] and thus yields a very efficient algorithm (see [3] for time results). On the contrary one can minimize a binary problem $E(11 . \leq \lambda \mid v)$ via solving the TV problem in $\mathcal{L}_{r}$ as proposed in [2]. A classical approach to minimize TV is to solve its Euler-Lagrange equation via a partial differential equation (PDE).

The maximum flow approach is extremely fast for solving binary or TV optimization problems [3]. However its main drawback is that it requires to build the graph. And applying this approach might be impossible when one works with large images or volumes. On the contrary note that a PDE approach to solve a TV problems [2] does not require more memory that the image itself. Thus one can solve the PDE to fin d a global minimizer of the binary problem.

* J. Darbon is supported by ONR grant N000140710810, Tony Chan by ONR grant N00014-06-1-0345 and NSF grant DMS-0610079, and Selim Esedoglu by NSF grant DMS 0713767 E-mail: jerome@math.ucla.edu,

** sylvain.lefebvre@sophia.inria.fr,

*** tfchan@nsf.gov,

† esedoglu@umich.edu, 
Solving such a PDE takes much more time than a maximum flow. However, it is straightforward to implement such a PDE on a Graphic Processor Unit (GPU) that is known for its remarkable performances. The following table presents the number of iterations performed in 1s on a Centrino 2.3 Ghz CPU and a Nidia GeForce 8800 GTX GPU.

\begin{tabular}{llll}
\hline size & GPU & CPU & ratio \\
\hline $256^{2}$ & 7200 & 119 & 60 \\
$512^{2}$ & 4000 & 30 & 133 \\
$1024^{2}$ & 1118 & 5 & 223 \\
$2048^{2}$ & 285 & 1.15 & 285 \\
\hline
\end{tabular}

Such preliminary results suggest that the loss of speed induced by solving the PDE might by compensated by the use of GPU. The full study of this approach will be described in a forthcoming paper.

\section{References}

[1] Y. Boykov, O. Veksler, and R. Zabih. Fast approximate energy minimization via graph cuts. IEEE PAMI, 23(11), 1222-1239 (2001).

[2] T.F. Chan and S. Esedog̃lu. Aspect of total variation regularized $l^{1}$ function approximation. SIAM Applied Mathematics.

[3] J. Darbon and M. Sigelle. Image restoration with discrete constrained Total Variation part I: Fast and exact optimization. JMIV, 26(3), 261-276 (2006).

[4] D. Hochbaum. An efficient algorithm for image segmentation, Markov Rand om Fields and related problems. Journal of the ACM, 48(2), 686-701 (2001)

[5] J.P. Picard and H.D. Ratlif. Minimum cuts and related problem. Networks, 5, (1975). 